\title{
NOZZLE DESIGN AND ASSISTANT-GAS FLOW ANALYSIS IN THE CO2 LASER CUTTING
}

\author{
Jun Chen ${ }^{1}$, Lixin $\mathrm{Lu}^{2}$, Limin $\mathrm{Li}^{1}$, and Hui Zhao ${ }^{1}$ \\ 'CIMS center, Shanghai University, Shanghai,200072, China,Email:pedrorichter@163.com \\ ${ }^{2}$ Shanghai Feilo Co.,LTD,Shanghai,200050, China
}

\begin{abstract}
This paper investigates the nozzle design and assistant-gas analysis. By some experiments, we present important date and some conclusions about the significant parameters of the laser.
\end{abstract}

Key words: laser cutting, nozzle, kerf.

\section{INTRODUCTION}

Lasers are widely used in industry as cutting tools due to ultra flexibility of the cutting conditions, obtaining high quality end product, quick set up, non-mechanical contact between the workpiece and the tool, and small size of the heat affected zone.

In china, various lasers products are now widely used for material processing such as cutting $\&$ drilling, welding, surface treatment, ablation, and so on. $\mathrm{CO}_{2}$ lasers and their applications to the fine cutting of the steel plate seems to have reached completion for meeting the user's demands. However, $\mathrm{CO}_{2}$ lasers are continuously being improved so that they may become applicable to more effective and more precise material processing. High laser power, high laser-beam quality, and highly controlled powermode are the essential items for attaining the purser.

In a laser system, laser beam focuses on the surface of workpiece and molten it though lens; meanwhile the co-axially compressed gas puffs away the dross in this process. The laser beam moves above the workpiece relatively and forms a cutting kerf on it.

Please use the following format when citing this chapter:

Chen, Jun, Lu, Lixin, Li, Limin, Zhao, Hui, 2006, in International Federation for Information Processing (IFIP), Volume 207, Knowledge Enterprise: Intelligent Strategies In Product Design, Manufacturing, and Management, eds. K. Wang, Kovacs G., Wozny M., Fang M., (Boston: Springer), pp. 519-524. 
The nozzle is utmost important part of a laser machine. Besides focus the laser beam on the workpiece, it provides the assistant-gas for cutting process. The composition, pressure, speed of the assistant-gas is all the top important parameters to a cutting system.

\section{NOZZLE DESIGN AND ANALYSIS}

As laser systems are becoming more industry demanding, the need for developments in the area of monitoring, diagnosis, regulation and modeling becomes essential to achieve and maintain a high-quality cutting process. The design of nozzle in a laser cutting system, is significant to a laser machine, Especially it's assistant-gas will greatly affect cutting process and cutting quality. Assisting gas protects the surface from high temperature oxidation reactions while high temperature exothermic reactions take place when oxygen is used as assisting gas.

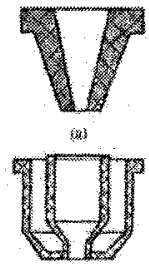

(1)

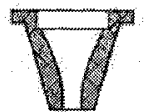

s?

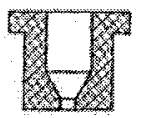

6
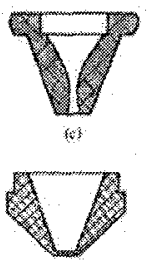

(1)

Figure 1. Different co-axial nozzle

The nozzle could be designed in different styles; fig1 presents some common co-axial nozzles. The diameter of the nozzle outlet should let the laser beam pass successfully and avoid touching the inner surface of the nozzle. In addition, the jet assistant-gas of the nozzle should couple to blow off the dross in the kerf and strength cutting effectively. Different styles of nozzle bring different pressure, velocity of the assistant-gas, and different cutting quality. If the diameter is too large, kerf width will be too wide, while it is too small, it will make the value of kerf inaccurate, diameter of the nozzle outlet is about $1 \sim 4 \mathrm{~mm}$.

\section{ASSISTANT-GAS ANALYSIS}

In general, it is impractical to investigate all of the factors at one time. The identification of the dominant factors for which variation could lead to 
large effects on the cut quality is important. The gas-composition variation, the gas pressure and the gas speed are the dominant factors; their effects on the cut quality need to be investigated.

As a co-axial nozzle system is used in laser cutting to assist the cutting process, the gas flow velocity at the nozzle exit is an important factor for the process. Because of the rapid change of pressure and temperature in nozzle, the wall surface is insufficient to transfer any appreciable amount of heat to the fluid. Therefore, the flow is considered adiabatic. Because of the short length of the nozzle and the smooth variation of its dimensions, the eddy effect and skin friction are neglected. Thus, the flow is considered as reversible and adiabatic, which means that the flow is isentropic.

The analysis associated with the influence of assisting gas including cooling and exothermic reaction contribution on the cutting process was examined in the previous study; therefore, the analysis will be described briefly. Assisting gas forms a boundary layer flow over the liquid surface (molten metal surface). The heat transfer from liquid surface to boundary laser flow occurs, since the assisting gas is at room temperature. Since the cooling effect of the assisting gas as well as melting and evaporation taking place at the laser irradiated surface are introduced in the scaling laws, only the exothermic reaction contribution of the assisting gas is introduced. Moreover, high temperature oxidation reaction (exothermic reaction during which metal oxides are formed) at the melt surface provides excess energy to the laser-irradiated region during the machining operation.

The species formed after the chemical reactions (product of exothermic reactions) contribute to the heat transfer-taking place at gas-liquid interface. In this case, heat transfer at the gas-liquid interface due to high temperature oxidation reaction can be described through the ratio of dimensionless heat transfer coefficients due to diffusion ( $\mathrm{CHd}$ ) and chemical reaction $(\mathrm{CHc})$, which was presented as (1):

$$
\frac{C_{H d}}{C_{H e}}=\frac{\left[1+B_{1}\left(u_{L} / u_{e}\right)\right]^{P_{\mathrm{r}-1}}}{\left[1+B_{1}\left(u_{L} / u_{e}\right)\right]^{S \mathrm{r}-1}}, B_{1}=\frac{2\left(\rho u_{e}\right)_{g}}{\rho_{e} u_{e} C_{f}}
$$

And Pr and Sc are turbulent Prandtl and Schmidt numbers, respectively. Ue is the gas jet velocity at the edge of the boundary layer. UL the liquid velocity, and $\mathrm{Cf}$ the skin friction coefficient. The rate of heat transfer ( $q$ LJC) per unit area (Kerf width $\times$ Kerf depth) of the molten metal excluding the cooling effect while including chemical reaction contribution of the assisting gas can be written as: 


$$
\dot{q} L J C=\rho_{e} u_{e} C_{H_{c}}\left[\left(\frac{C_{H d}}{C_{H e}}-1\right) h_{c}\right]
$$

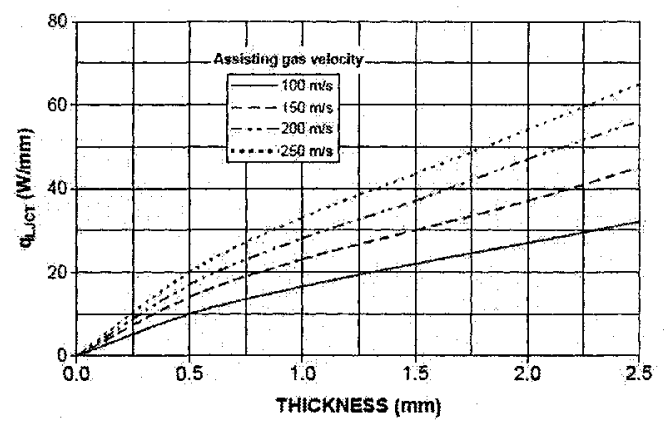

Figure 2.digram of different velocity

The quantity $q$ LJC was computed for various Kerf depths as shown in Fig2; therefore, the values of $q$ LJC computed in the previous study are accommodated in the analysis. Moreover, the quantity $q$ LJC can be integrated over the cut width to obtain the rate of chemical energy contribution during the cutting process. Fig2 shows graphs of four assisting gas velocity, as we can see, the $q$ LJC in a certain thickness is increasing with the rising of velocity.

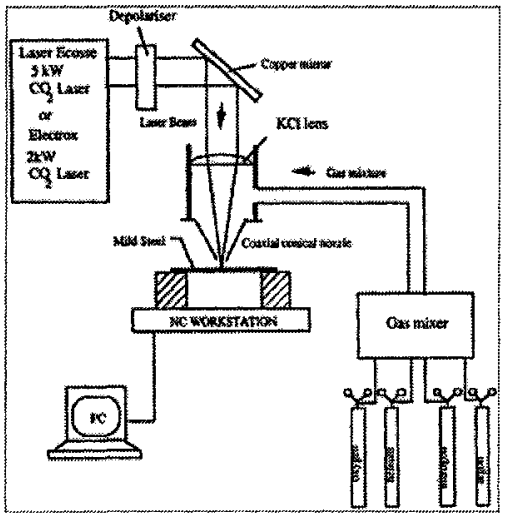

Figure 3.model of different gas composition

Through investigation of how the composition of the assistant-gas affecting the cutting process, a model can be set up in Fig3.to exemplify this. 
If the gas flow condition inside the kerf during the cutting process is considered similar to pope flow, the gas flow from the nozzle is uniform with a free stream velocity at the slot entrance. As it moves down the slot, the friction between the cut wall and the flow stream element will generate a shear force to retard the flow motion and cause the boundary layer to grow until it is fully developed. For laminar flow, the Length (entrance length) required for the velocity to be unchanged with axial position can be approximated to $(0.05)^{*} \operatorname{Re}^{*} D$, where $\operatorname{Re}$ is the Reynolds number and $D$ is the diameter of the pipe.

From the table1, it is easy to see that the shear force on the cutting front is influenced largely by the gas flow velocity and the density. Increasing the gas flow velocity will significantly increase the shear drag force on the cutting front. High density has higher shear drag force, thus from tablel argon may be the most promising gas for $\mathrm{CO}_{2}$ lasers cutting and helium the least promising.

Table 1: date of different gas parameters

\begin{tabular}{lllll}
\hline \multirow{2}{*}{ Parameter } & \multicolumn{3}{c}{ Gas } \\
\cline { 2 - 5 } & Oxygen & Argon & Helium & Nitrogen \\
\hline$\rho:$ Density $\left(\mathrm{kg} \mathrm{m}^{-3}\right)$ & 1.2997 & 1.8435 & 0.1779 & 1.1367 \\
$\mu$ :dynamic viscosity $\left(106 \mathrm{~N} \mathrm{~s} \mathrm{~m}^{-2}\right)$ & 2.0854 & 2.2693 & 1.9618 & 1.8003 \\
$v$ :kinematics' viscosity $\left(10^{5} \mathrm{~m}^{2} \mathrm{~s}^{-1}\right)$ & 1.5498 & 1.2734 & 11.1007 & 1.5498 \\
$C_{P}:$ specific heat of constant pressure $\left(\mathrm{kJ} \mathrm{K}^{*}\right.$ & 0.9213 & 0.5198 & 5.2015 & 1.0436 \\
$\left.{ }^{1} \mathrm{~kg}^{-1}\right)$ & & & & \\
$k^{*}:$ thermal conductivity $\left(10^{2} \mathrm{~W}^{-1} \mathrm{~K}^{-1} \mathrm{~m}^{-1}\right)$ & 2.6735 & 1.7637 & 15.3879 & 2.5768 \\
\hline
\end{tabular}

We also obtained table 2 and Fig4 by investigating the pressure of the assistant-gas. These experiments were performed with $2.0 \mathrm{~kW} \mathrm{CO} 2$ laser, the laser mode structure being TEM01. The new cutting head was used in these high-pressure cutting experiments. Four different assistant gases used in this study, being pure oxygen, pure Argon, pure nitrogen, and air (78\% nitrogen and $22 \%$ oxygen). The first three types of gases are commercial standard gases, whilst the air used in the experiments is supplied by an air compressor through an air cleaner. The cutting experiments were carried out primarily on $3 \mathrm{~mm}$ thick mild steel plate.

The influence of the cutting speed by the gas pressure can be seen by table2, for a certain assistant-gas, increase the pressure can improve the cutting speed obviously. The Fig4 tells us the different cutting speed and pressure is significant to the kerf quality. 


\begin{tabular}{lllll}
\hline mm s & 2 bar & 4 bat & 6 bar & 10 bar \\
\hline Orygen & $52.5 \pm 2.5$ & $57.5 \pm 2.5$ & $60.5 \pm 2.5$ & $110 \pm 2.5$ \\
Nitrogen & $17.5 \pm 2.5$ & $17.5 \pm 2.5$ & $17.5 \pm 2.5$ & $22.5 \pm 2.5$ \\
Argon & $17.5 \pm 2.5$ & $17.5 \pm 2.5$ & $17.5 \pm 2.5$ & $27.5 \pm 2.5$ \\
Air & $22.5 \pm 2.5$ & $27.5 \pm 2.5$ & $37.5 \pm 2.5$ &
\end{tabular}

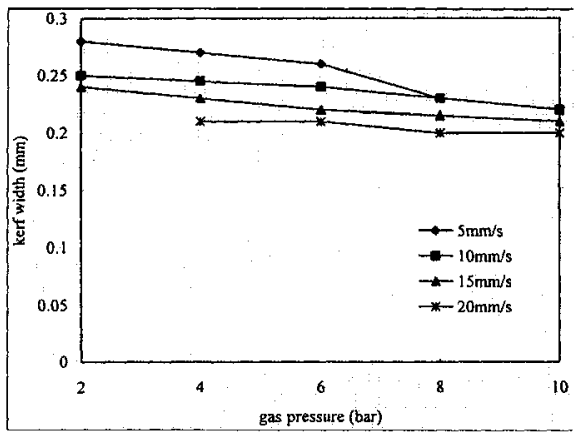

Figure 4. kerf in different gas presure

\section{CONCLUSIONS}

We investigate nozzle design and gas analysis by some experiments. These research show, the design of nozzle, composition, velocity, pressure of assistant-gas, will affect greatly in the cutting process.

\section{REFERENCE}

[1] K.A. Bunting, G. Cornfield, Toward a general theory of cutting: a relationship between the incident power density and the cut speed, ASME J. Heat Transfer 97 (1975) 116-121.

[2] K. Danisman, B.S. Yilbas, Z. Yilbas, Study of some characteristics of plasma generated during CO2 laser beam cutting process, Opt. Laser Technol. 24 (1992) 33-38.

[3] S.L. Chen, The effects of gas composition on the $\mathrm{CO} 2$ laser cutting of mild steel, J. Mater. Process. Technol. 73 (1998) 234-242. 89-95.

[4] M.J. Hsu, P.A. Molian, Off-axis, gas-jet-assisted, laser cutting of $6.35 \mathrm{~mm}$ thick stainless steel, ASME J. Eng. Ind. 117 (1995) 272- 276.

[5] B.S. Yilbas, A.Z. Sahin, Turbulent boundary layer approach allowing chemical reactions for CO2 laser oxygen-assisted cutting process, Proc. Inst. Mech. Eng. C 208 (1994) 275284.

[6] B.S. Yilbas, The analysis of CO2 laser cutting, Proc. Inst. Mech. Eng. B 211 (1997)

[7] B.S. Yilbas, A study into laser cutting process, Heat Mass Transfer 32 (1997) 175-180. 